\title{
Dasatinib and chemotherapy in a patient with early T-cell precursor acute lymphoblastic leukemia and NUP214-ABL1 fusion: A case report
}

\author{
YAN CHEN $^{1 *}$, LI ZHANG $^{2 *}$, JIANKUN HUANG $^{3}$, XIULI HONG ${ }^{1}$, \\ JIANGNING ZHAO $^{1}$, ZHAO WANG ${ }^{1}$ and KEJIE ZHANG ${ }^{1}$
}

\author{
${ }^{1}$ Department of Hematology, Zhongshan Hospital, Xiamen University, Xiamen, Fujian 361004; \\ ${ }^{2}$ Department of Hematology, West China Hospital, Sichuan University, Chengdu, Sichuan 610041; \\ ${ }^{3}$ Central Laboratory, Zhongshan Hospital, Xiamen University, Xiamen, Fujian 361004, P.R. China
}

Received February 26, 2016; Accepted March 23, 2017

DOI: 10.3892/etm.2017.5046

\begin{abstract}
The present study aimed to evaluate the therapeutic efficacy of dasatinib in a patient with nucleoporin 214-tyrosine protein kinase ABL1 proto-oncogene 1 (NUP214-ABL1)-positive early T-cell precursor-acute lymphoblastic leukemia (ETP-ALL), as well as that of selinexor and dasatinib for NUP214-ABL1-positive ETP-ALL in vitro. ETP leukemia is a form of T-cell ALL (T-ALL) with poor prognosis. The NUP214-ABL1 gene is present in $\sim 6 \%$ of T-ALL cases, however the prevalence of NUP214-ABL1 gene expression in ETP-ALL in particular has not yet been verified. The current study reports the rare case of a 29-year-old man with ETP-ALL harboring the NUP214-ABL1 fusion gene, presenting with low-grade fever, stomachache and splenomegaly. The patient was successfully treated with dasatinib and vincristine, idarubicin, cyclophosphamide and prednisone (VICP) chemotherapy. The therapeutic efficacy of selinexor and dasatinib was also evaluated in vitro. Apoptosis was analyzed using Annexin V/propidium iodide staining and flow cytometry, and poly ADP-ribose polymerase (PARP) cleavage was detected using western blot analysis. The results demonstrated that the apoptotic cell population significantly increased following selinexor or dasatinib treatment compared with the control $(\mathrm{P}<0.05)$. Furthermore, combined selinexor and dasatinib treatment led to a significant increase in cell apoptosis compared with either treatment alone $(\mathrm{P}<0.05)$. The apoptosis results
\end{abstract}

Correspondence to: Dr Kejie Zhang, Department of Hematology, Zhongshan Hospital, Xiamen University, 201-209 Hubinnan Road, Xiamen, Fujian 361004, P.R. China

E-mail: zhangkejie0378@sina.com

*Contributed equally

Key words: early T-cell precursor acute lymphoblastic leukemia, NUP214-ABL1, dasatinib, selinexor, T-cell were confirmed by PARP cleavage. Thus, NUP214-ABL1 fusion gene expression should be tested in T-ALL, including ETP-ALL. Dasatinib used in combination with traditional induction chemotherapy may reverse the high induction failure of ETP-ALL with NUP214-ABL1 fusion gene; however, further prospective studies are required to confirm this. Therefore, selinexor with or without dasatinib may serve as a potential salvage therapy in the case of relapse and may be developed as a novel treatment for ETP-ALL with the NUP214-ABL1 fusion gene.

\section{Introduction}

Early T-cell precursor-acute lymphoblastic leukemia (ETP-ALL) has been identified as a distinct biological subtype of T-cell lineage ALL (T-ALL), which accounts for $15 \%$ of T-ALL cases (and $2 \%$ of ALL cases). It is associated with poor clinical outcomes even following the use of current treatment regimens. Recently, the complete remission rate/complete remission with incomplete platelet recovery rate in patients with ETP-ALL was indicated to be significantly lower than that of non-ETP-ALL patients ( 73 vs. $91 \% ; \mathrm{P}=0.03$ ) in 2016 (1). Up to the last follow up, the median overall survival for patients with ETP-ALL was 20 months vs. not yet reached for the non-ETP-ALL/LBL patients $(\mathrm{P}=0.008)(1)$. This subtype is characterized by a lack of expression of the T-cell surface markers cluster of differentiation (CD) 1a and CD8, weak or absent expression of CD5, and aberrant expression of at least one of the following myeloid or hematopoietic stem cell markers: CD13, CD33, CD34, CD117, human leukocyte antigen (HLA)-DR, CD11b and CD65 (2).

T-ALL is a genetically heterogeneous disease caused by the accumulation of multiple genetic defects in developing T-cells that affect critical cellular processes, including cell differentiation, proliferation, survival and self-renewal capacity (3). The presence of the nucleoporin 214-ABL proto-oncogene 1 (NUP214-ABL1) fusion gene has been identified in $6 \%$ of all T-ALL cases (4). NUP214, also known as CAN, is a $214-\mathrm{kDa}$ FG-repeat-containing nucleoporin located at band $9 \mathrm{q} 34.1$ and includes 36 exons. The 
encoded protein is located at the cytoplasmic side of the nuclear pore complex (NPC) (5). The NUP214-ABL1 fusion protein is a constitutively active protein tyrosine kinase. De Keersmaecker et al (6) recently identified that the sparse representation-based classifier (SRC) family kinase lymphocyte-specific protein tyrosine kinase (LCK) is a protein essential for the proliferation and survival of T-ALL cells dependent on NUP214-ABL1 activity. These findings underscore the potential of the dual ABL1/SRC kinase inhibitor dasatinib in the treatment of NUP214-ABL1-positive T-ALL. Furthermore, NUP214 serves a role in mRNA export and chromosome region maintenance 1 (CRM1)-mediated export of the 60S pre-ribosomal subunit, and may serve a role in the transport of other proteins (7). Given that CRM1 inhibitors, such as selinexor, suppress the export of proteins associated with NUP214 (7), there is potential for their use to treat patients with T-ALL that also harbor NUP214-ABL1.

The NUP214-ABL1 gene is highly specific for T-ALL (8), however the prevalence of NUP214-ABL1 gene expression in ETP-ALL has not been verified. Zhang et al (9) reported that NUP214-ABL1 was not detected in all the 64 ETP-ALL cases studied. The present case report describes a rare case of a patient with ETP-ALL harboring the NUP214-ABL1 fusion gene. The present study also evaluated the therapeutic efficiency of selinexor and dasatinib to treat NUP214-ABL1-positive ETP-ALL cells in vitro. ETP-ALL has higher rates of relapse and remission failure following conventional chemotherapy and shorter patient survival times (1), meaning that novel therapeutic agents to treat ETP-ALL are urgently required.

\section{Case report}

A 29-year-old Han Chinese man was admitted to the Zhongshan Hospital, Xiamen University (Xiamen, China) in September 2015 with a low-grade fever and stomachache, which he had experienced for 5 days. The patient had no family history of genetic or hematological disease. Ethical approval for the current case report was granted by the Medical Ethics Committee of Zhongshan Hospital, Xianmen University (Xiamen, China) and informed written consent was obtained from the patient.

The patient presented with splenomegaly (spleen size, $13.2 \times 4.3 \mathrm{~cm}$ ) determined via abdominal ultrasonography. The patient's peripheral blood was analyzed with a hematology analyzer (Beckman Coulter, Inc., Brea, CA, USA): White blood cell count was $5.86 \times 10^{9} / 1$ (neutrophils, $10 \%$; lymphocytes, 66\%; monocytes, 20\%; reference range, white blood cell count $3.5 \times 10^{9} / 1-9.5 \times 10^{9} / 1$; neutrophils, $50-70 \%$; lymphocytes, 20-50\%; monocytes, 3-10\%), hemoglobin level ( $\mathrm{Hb}$ ) was $118 \mathrm{~g} / 1$ (reference range, 130-175 g/l) and blood platelet count (Plat) was $45 \times 10^{9} / 1$ (reference range, $\left.125 \times 10^{9} / 1-350 \times 10^{9} / 1\right)$. The proportion of immature cells detected in the peripheral blood was low (4\%). The following blood coagulation characteristics were measured: Prothrombin time, $15.6 \mathrm{sec}$ (reference range, 11.0-15.0 sec); activated partial thromboplastin time, $15.2 \mathrm{sec}$ (reference range, 28.0-42.5 sec); fibrinogen level, $2.82 \mathrm{~g} / 1$ (reference range, 2.00-4.00 g/l) and D-dimer quantification, $3,150 \mathrm{ng} / \mathrm{ml}$ (reference range, $0.00-500.00 \mathrm{ng} / \mathrm{ml}$. Lactate dehydrogenase levels were elevated (605.1 U/1; reference range, 109.0-245.0 U/l), however liver and renal functions were
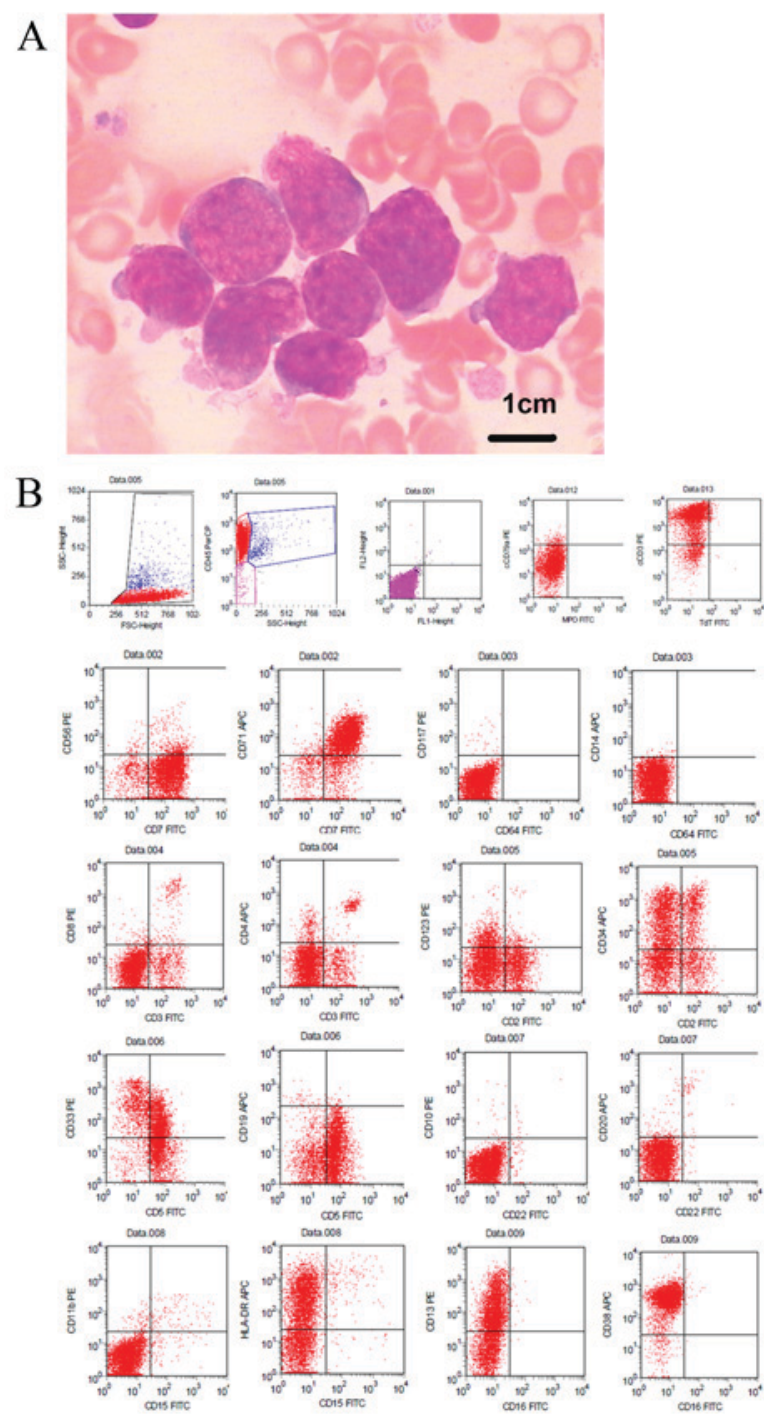

Figure 1. Histomorphological features of leukemic blasts from bone marrow aspirates and flow cytometry analysis. (A) Blasts were large and had high nuclear/cytoplasm ratios, irregular nuclei, dispersed chromatin pattern, indistinct nucleoli and basophilic cytoplasm with pseudopods (Wright-Giemsa stain, the white blood cell nucleus and cytoplasm are indicated by the characteristic blue or pink coloration; magnification, x1,000). (B) Flow cytometric immunophenotyping.

normal. The patient underwent bone marrow (BM) harvesting under general anesthesia. BM was aspirated from the posterior iliac crest in a sterile operating room, and BM smears stained with Wright-Giemsa stains (Electron Microscopy Sciences, Hatfield, PA, USA) were obtained and tested under an optical microscope. The white blood cell nucleus and cytoplasm were indicated by the characteristic blue or pink coloration. The 500-cell differential count from the BM aspirate on the first day of hospital admission revealed that $80 \%$ of all nucleated cells were blasts. The majority of blasts exhibited large sizes, high nuclear/cytoplasm ratios, irregularly shaped nuclei, dispersed chromatin, indistinct nucleoli and basophilic cytoplasm that occasionally formed pseudopods, but few exhibited moderate amounts of granular cytoplasm, fine nuclear chromatin and 2-3 prominent nucleoli (Fig. 1A). Cytochemical staining (10) was negative for myeloperoxidase (MPO) and $\sim 3 \%$ positive for periodic acid-Schiff staining. 
Table I. Genetic analysis of the early T-cell precursor-acute lymphoblastic leukemia patient.

\begin{tabular}{|c|c|c|c|c|c|c|c|}
\hline & \multirow{2}{*}{ Type of analysis } & \multirow{2}{*}{ Gene } & \multirow{2}{*}{ Method } & \multirow{2}{*}{ Result } \\
\hline & & & & & & & \\
\hline Type of analysis & Gene & Method & Result & \multirow{16}{*}{ Gene mutation } & & \multirow{16}{*}{$\begin{array}{l}\text { RT-PCR } \\
\text { Next- } \\
\text { generation } \\
\text { sequencing }\end{array}$} & \\
\hline Gene mutation & FLT3-ITD & RT-PCR & - & & $\begin{array}{l}\text { FLI3-ITD } \\
\text { IDH1 }^{\mathrm{R} 132}\end{array}$ & & $\begin{array}{l}- \\
-\end{array}$ \\
\hline \multirow[t]{15}{*}{ Fusion gene } & MLL/AF4 & $\begin{array}{l}\text { Multiplex } \\
\text { nested }\end{array}$ & - & & & & \\
\hline & & RT-PCR & & & NPM1(exon12) & & - \\
\hline & MLL/AF6 & & - & & IDH2R140 & & - \\
\hline & MLL/AF9 & & - & & CEBPA & & - \\
\hline & MLL/AF10 & & - & & IDH2R172 & & - \\
\hline & MLL/AF17 & & - & & DNMT3A & & - \\
\hline & MLL/AF1P & & - & & C-kitD816 & & - \\
\hline & MLL/AF1Q & & - & & PHF6 & & - \\
\hline & MLL/AFX & & - & & TET2 & & (c.86C>G; \\
\hline & MLL/ELL & & - & & & & p.P29R) \\
\hline & MLL/ENL & & - & & ASXL1 & & (c. $1954 \mathrm{G}>\mathrm{A}$; \\
\hline & dupMLL & & - & & & & p.G652S), \\
\hline & CBF $\beta / M Y H 11$ & & - & & & & (c.3759T >C; \\
\hline & AML1/ETO & & - & & & & p.S1253S) \\
\hline & AML1/MDS1 & & - & & & & \\
\hline
\end{tabular}

SET/CAN

DEK/CAN

PML/RAR $\alpha$

PLZF/RAR $\alpha$

NPM/RAR $\alpha$

NPM/ALK

TEL/AML1

E2A/PBX1

$\mathrm{BCR} / \mathrm{ABL} 1$

NPM/MLF1

TEL/ABL1

E2A/HLF

TLS/ERG

SIL/TAL1

TEL/PDGFR

EVI1

HOX11

Ph-like fusion genes
Table I. Continued.

RT-PCR, reverse transcription-polymerase chain reaction; +, positive; -, negative; Ph-like, Philadelphia chromosome-like.

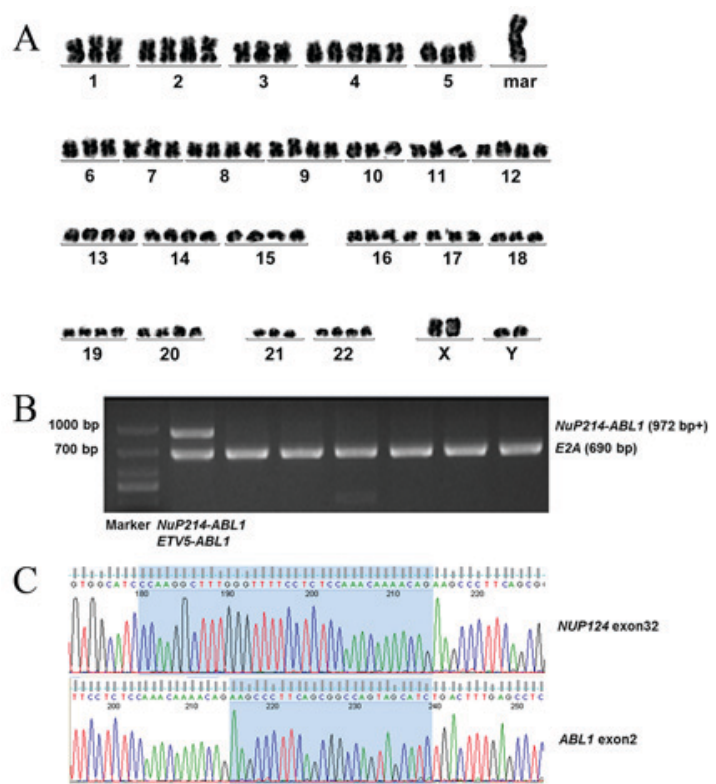

Figure 2. Karyotype of the abnormal clone and detection of the NUP214-ABL1 fusion transcript. (A) Karyotype of the abnormal clone. The final karyotype was $80-83<4 \mathrm{n}>$,XXYY,+ mar, inc [2]/46, XY[18]. (B) NUP214-ABL1 fusion transcript detected by multiplex reverse transcription-polymerase chain reaction. (C) NUP214-ABL1 fusion transcript detected by DNA sequencing. NUP124 exon 32 and $A B L 1$ exon 2 are shaded blue. NUP214-ABL1, nucleoporin 214-tyrosine protein kinase ABL1 proto-oncogene 1.

Flow cytometry of the BM aspirate was performed using a four-color Beckman Coulter Cytomics FC 500 flow cytometer (Beckman Coulter, Inc.) as described previously (11). The results indicated that $\sim 90 \%$ of the blasts were positive for CD45, the T-cell markers cCD3, CD2 and CD7, and the myeloid/stem 
A

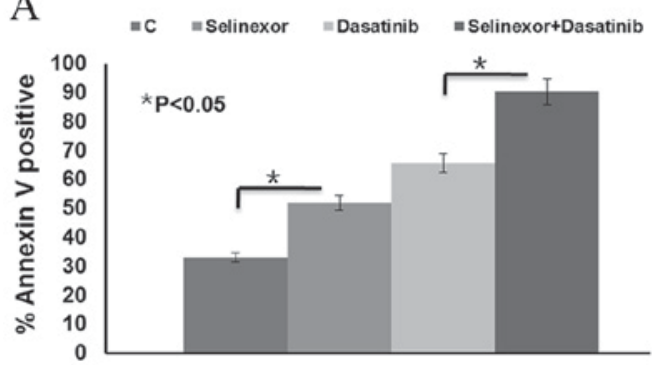

B

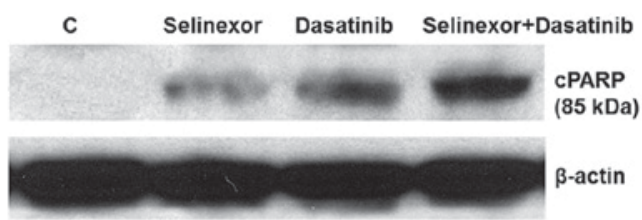

Figure 3. Effect of selinexor and dasatinib treatment in primary biopsy-derived NUP214-ABL1-positive lymphoblast cells. Freshly isolated lymphoblast cells were treated with $10 \mu \mathrm{M}$ selinexor and/or $10 \mu \mathrm{M}$ dasatinib for $48 \mathrm{~h}$. (A) Apoptosis was measured using Annexin V/propidium iodide staining and flow cytometry. (B) Cell extracts were analyzed by western blot analysis to determine PARP cleavage. PARP, poly ADP-ribose polymerase; C, control; NUP214-ABL1, nucleoporin 214-tyrosine protein kinase ABL1 proto-oncogene 1.

cell markers CD34, CD38, CD13, CD33, HLA-DR and CD123. However, the blasts were weak for CD5, negative for CD1a, $\mathrm{CD} 8$, as well as CD19, CD10, CD79a, terminal deoxynucleotidyl transferase, CD4, CD3, CD64, CD14, CD20, CD56, CD16, CD15, CD11b and MPO (Fig. 1B), suggesting a diagnosis of ETP-ALL. Bone marrow cells were directly processed for chromosomal preparation and cytogenetic analyses were performed using the standard method and/or the G-banding method (12). The result of conventional G-banding chromosomal analysis of the BM cells was $80-83<4 \mathrm{n}>, \mathrm{XXYY},+$ mar,i nc[2]/46,XY[18] (Fig. 2A).

The results of multiplex nested reverse transcriptionpolymerase chain reaction (RT-PCR), which was performed as described previously (13), were negative for the fusion genes listed in Table I. Multiplex RT-PCR was subsequently used to detect Philadelphia chromosome-like fusion genes that have a gene expression profile similar to that of $\mathrm{Ph}^{+}$ ALL, but lack $B C R A B L 1$ fusion. The results demonstrated that the NUP214-ABL1 fusion gene was positive in this case (Table I; Fig. 2B), which was further confirmed by DNA sequencing (Fig. 2C). Also, gene mutation analyses (Table I) were performed. A TET2 missense mutation (c.86C $>$ G p.P29R), ASXL1 missense mutation (c.1954G >A; p.G652S) and ASXL1 same-sense mutation (c.3759T>C; p.S1253S) were detected in this case, which were single nucleotide polymorphism variants (SNP) of TET2 and ASXL1 mutations. These SNP variants did not suggest any significant prognostic impact on leukemia.

The patient received pretreatment with an intravenous drip of $10 \mathrm{mg} /$ day dexamethasone (Cisen Pharmaceutical Co., Ltd., Shandong, China). Following treatment for 7 days, stomachache and fever were attenuated and the patient's lymphocyte count decreased to $<1.0 \times 10^{9} / 1$. The patient subsequently received induction chemotherapy with vincristine (Hangzhou Minsheng Pharmaceutical Group Co., Ltd., Hangzhou, China), idarubicin (Pfizer Inc., New York, NY, USA), cyclophosphamide (Baxter Oncology GmbH, Shanghai, China) and prednisone (Cisen Pharmaceutical Co., Ltd.) (VICP; $2 \mathrm{mg}$ vincristine on days 1, 8, 15 and 22; $10 \mathrm{mg}$ idarubicinon days 1-3 and days 15-17; $1.2 \mathrm{~g}$ cyclophosphamide on days 1 and 15; and $60 \mathrm{mg}$ prednisone on days 1-28) and dasatinib (100 mg/day, on days 8-14). On day 15 , routine blood examination results were as follows: Neutrophils, 4.76x 10 $/ 1$; lymphocytes, $1.85 \times 10^{9} / 1, \mathrm{Hb} 65 \mathrm{~g} / 1$ and Plat $74 \times 10^{9} / 1$ (reference range, neutrophils, $1.8 \times 10^{9} / 1-6.3 \times 10^{9} / 1$; lymphocytes, $1.10 \times 10^{9} / 1-3.2 \times 10^{9} / 1, \mathrm{Hb} 130-175 \mathrm{~g} / 1$ and Plat $\left.125 \times 10^{9} / 1-350 \times 10^{9} / 1\right)$. BM aspiration revealed $<5 \%$ lymphoblasts, indicating that the patient had achieved a complete hematological remission with incomplete blood count recovery. On day 30, the patient had achieved complete hematological remission (CR), which was defined as $<5 \%$ marrow blasts in the bone marrow aspirate and normalization of blood counts. The patient then received consolidation chemotherapy $[1.2 \mathrm{~g}$ cyclophosphamide on day 1; 3 g cytarabine (Pfizer Inc.) every $12 \mathrm{~h}$ on days 1-2; and $80 \mathrm{mg}$ 6-mercaptopurine (Hangzhou Minsheng Pharmaceutical Group Co., Ltd.) on days 1-7] and adequate central nervous system prophylaxis with intrathecal therapy [10 mg methotrexate (Pfizer Inc.), $50 \mathrm{mg}$ cytarabine and $10 \mathrm{mg}$ dexamethasone] and high-dose systemic chemotherapy with $5 \mathrm{~g} / \mathrm{m}^{2}$ methotrexate. The final date of follow-up was January 11 2016, at which point the patient was alive and healthy.

In vitro analysis of selinexor and dasatinib treatment. Upon written informed consent, in vitro analysis was conducted on cells from the patient. A total of $2 \times 10^{6}$ primary lymphoblast cells were obtained from BM samples using Ficoll density gradient centrifugation (FicollPaque PLUS solution; GE Healthcare Life Sciences, Little Chalfont, UK). The protocol was as follows: $4 \mathrm{ml}$ of Ficoll Histopaque was placed in a $15 \mathrm{ml}$ centrifuge tube, then the bone marrow sample was gently layered on top. The tubes were centrifuged for $30 \mathrm{~min}$ at $100 \mathrm{xg}$ at $4^{\circ} \mathrm{C}$ in a swing-out bucket. The buffy coat that formed in the interphase between histopaque and medium was aspirated and washed (centrifuged at $100 \mathrm{x}$ g for $10 \mathrm{~min}$ ) twice with $10 \mathrm{ml}$ of sterile PBS. Primary lymphoblast cells were maintained in RPMI 1640 medium (Thermo Fisher Scientific, Inc.) and 15\% fetal calf serum (HyClone; GE Healthcare Life Sciences, Logan, UT, USA), and incubated in an atmosphere containing $5 \% \mathrm{CO}_{2}$ at $37^{\circ} \mathrm{C}$. The effect of selinexor and dasatinib on apoptosis was evaluated in primary lymphoblast cells. The primary cells were treated with $10 \mu \mathrm{M}$ selinexor (14) and/or $10 \mu \mathrm{M}$ dasatinib (15) for $48 \mathrm{~h}$ at $37^{\circ} \mathrm{C}$. Apoptosis was subsequently analyzed using the Annexin V/propidium iodide staining (BD PharMingen, San Diego, CA, USA) and flow cytometry was performed according to the manufacturer's protocol. Poly ADP-ribose polymerase (PARP) cleavage was detected using western blot analysis (16). 
The results demonstrated that the apoptotic cell population significantly increased following selinexor or dasatinib treatment compared with the control $(\mathrm{P}<0.05)$ and combined selinexor and dasatinib treatment led to a significant increase in cell apoptosis compared with either treatment alone $(\mathrm{P}<0.05$; Fig. 3A). These results were further confirmed by the detection of increased PARP cleavage by western blot analysis following selinexor or dasatinib treatment, compared with the control (Fig. 3B). Combined selinexor and dasatinib treatment induced more PARP cleavage than either treatment alone.

\section{Discussion}

Approximately $8 \%$ of T-ALL cases harbor fusions involving the $A B L 1$ tyrosine kinase gene (17). BCR-ABL1, the prototypic ABL1 fusion kinase in chronic myeloid leukemia and subsets of B-cell ALL, is rarely detected in T-ALL (18). By contrast, $6 \%$ of T-ALL cases express the constitutively active NUP214-ABL1 fusion kinase (5). To the best of our knowledge, there have been no studies reporting NUP214-ABL1 fusion gene expression in ETP-ALL to date. The current case report describes a rare case of ETP-ALL with NUP214-ABL1 fusion gene expression. ETP-ALL is recognized as a form of T-ALL with high induction failure (1). However, CR was achieved in the current case following 30 days induction therapy with VICP and dasatinib. Further studies are required to confirm that dasatinib used in combination with traditional induction chemotherapy is more effective than chemotherapy alone.

The NUP214-ABL1 fusion protein is sensitive to tyrosine kinase inhibitors including imatinib, dasatinib and nilotinib (17); therefore, these drugs may have potential in treating this subgroup of patients with T-ALL. Although NUP214-ABL1 is sensitive to ABL1 kinase inhibitors, the development of resistance to these compounds is a major clinical problem, underlining the requirement for additional drug targets in the sparsely studied NUP214-ABL1 signaling network. De Keersmaecker et al (6) identified LCK, mitotic arrest deficient-like 1, structural maintenance of chromosomes protein 4 and nucleoporin 155 as proteins that NUP214-ABL1-positive T-ALL tumor cells depend on for their proliferation, indicating that these proteins are potential drug targets for NUP214-ABL1-positive T-ALL. Targeting LCK in NUP214-ABL1 may be possible to treat patients with T-ALL that are NUP214-ABL1-positive, as dasatinib and bosutinib are able to co-target ABL1 and LCK (6). The effect of dasatinib on the apoptosis of primary isolated lymphoblast cells from the patient in the present case study was evaluated. Following treatment for $48 \mathrm{~h}$, apoptosis was induced, indicating that dasatinib induces a cytotoxic effect in NUP214-ABL1-positive cells.

Furthermore, De Keersmaecker et al (8) reported that incorporating NUP214-ABL1 into the NPC may be critical for the activation of NUP214-ABL1 kinase. NUP214 is one component of NPC proteins. Evolutionarily conserved NPC proteins mediate the transport of molecules between the nucleoplasm and cytoplasm, which are CRM1 dependent. Mutations in nucleoporins are often linked to specific developmental defects and disease, and the resulting phenotypes are usually interpreted as the consequences of perturbed nuclear transport activity (8). The present study indicated that CRM1 inhibition with selinexor was effective against a case of NUP214-ABL1-positive T-ALL in vitro. Therefore, selinexor may have a potential function as a salvage therapy at the time of relapse.

In conclusion, expression of the NUP214-ABL1 fusion gene should be tested in cases of T-ALL, including ETP-ALL. Dasatinib in combination with traditional induction chemotherapy may reverse the high induction failure of ETP-ALL with NUP214-ABL1 fusion gene, although further prospective studies are required to confirm this. Therefore, selinexor with or without dasatinib may serve as a potential salvage therapy following relapse.

\section{References}

1. Jain N, Lamb AV, O'Brien S, Ravandi F, Konopleva M, Jabbour E, Zuo Z, Jorgensen J, Lin P, Pierce S, et al: Early T-cell precursor acute lymphoblastic leukemia/lymphoma (ETP-ALL/LBL) in adolescents and adults: A high-risk subtype. Blood 127: 1863-1869, 2016.

2. Coustan-Smith E, Mullighan CG, Onciu M, Behm FG, Raimondi SC, Pei D, Cheng C, Su X, Rubnitz JE, Basso G, et al: Early T-cell precursor leukaemia: A subtype of very high-risk acute lymphoblastic leukaemia. Lancet Oncol 10: 147-156, 2009.

3. De Keersmaecker K, Marynen P and Cools J: Genetic insights in the pathogenesis of $\mathrm{T}$ cell acute lymphoblastic leukemia. Haematologica 90: 1116-1127, 2005.

4. Graux C, Cools J, Melotte C, Quentmeier H, Ferrando A, Levine R, Vermeesch JR, Stul M, Dutta B, Boeckx N, et al: Fusion of NUP214 to ABL1 on amplified episomes in T-cell acute lymphoblastic leukemia. Nat Genet 36: 1084-1089, 2004.

5. Pante N, Bastos R, McMorrow I, Burke B and Aebi U: Interactions and three-dimensional localization of a group of nuclear pore complex proteins. J Cell Biol 126: 603-617, 1994.

6. De Keersmaecker K, Porcu M, Cox L, Girardi T, Vandepoel R, de Beeck JO, Gielen O, Mentens N, Bennett KL and Hantschel O: NUP214-ABL1-mediated cell proliferation in T-cell acute lymphoblastic leukemia is dependent on the LCK kinase and various interacting proteins. Haematologica 99: 85-93, 2014.

7. Hutten S and Kehlenbach RH: Nup214 is required for CRM1-dependent nuclear protein export in vivo. Mol Cell Biol 26: 6772-6785, 2006.

8. De Keersmaecker K, Rocnik JL, Bernad R, Lee BH, Leeman D, Gielen O, Verachtert H, Folens C, Munck S, Marynen P, et al: Kinase activation and transformation by NUP214-ABL1 is dependent on the context of the nuclear pore. Mol Cell 31: 134-142, 2008.

9. Zhang J, Ding L, Holmfeldt L, Wu G, Heatley SL, Payne-Turner D, Easton J, Chen X, Wang J, Rusch M, et al: The genetic basis of early T-cell precursor acute lymphoblastic leukaemia. Nature 481: 157-163, 2012.

10. Leinonen EA: Cytochemical studies of acute leukemias. Acta Haematol 43: 219-227, 1970.

11. Tamul KR, Meyers DC, Bentley SA and Folds JD: Two color flow cytometric analysis of concomitant acute myeloid leukemia and chronic lymphocytic leukemia. Cytometry 18: 30-34, 1994.

12. Summer AT, Evans HJ and Buckland RA: New technique for distinguishing human chromosomes. Nature 232: 31-32, 1971.

13. Pallisgaard N, Hokland P, Riish øj DC, Pedersen B and Jørgensen P: Multiplex reverse transcription-polymerase chain reaction for simultaneous screening of 29 translocations and chromosomal aberrations in acute leukemia. Blood 92: 574-588, 2014.

14. Etchin J, Sanda T, Mansour MR, Kentsis A, Montero J, Le BT, Christie AL, McCauley D, Rodig SJ, Kauffman M, et al: KPT-330 inhibitor of CRM1 (XPO1)-mediated nuclear export has selective anti-leukaemic activity in preclinical models of T-cell acute lymphoblastic leukaemia and acute myeloid leukaemia. Br J Haematol 161: 117-127, 2013. 
15. Quintás-Cardama A, Tong W, Manshouri T, Vega F, Lennon PA, Cools J, Gilliland DG, Lee F, Cortes J, Kantarjian H, et al: Activity of tyrosine kinase inhibitors against human NUP214-ABL1-positive T cell malignancies. Leukemia 22: 1117-1124, 2008.

16. Zhang YL, Guang MH, Zhuo HQ, Min XH, Yao Q, Gu AQ, Wu SH, Zhang DB, Lu JY, Chen Y, et al: Carfilzomib inhibits constitutive $\mathrm{NF}-\kappa \mathrm{B}$ activation in mantle cell lymphoma $\mathrm{B}$ cells and leads to the induction of apoptosis. Acta Haematol 137: 106-112, 2017.
17. De Keersmaecker K, Marynen P and Cools J: Genetic insights in the pathogenesis of T-cell acute lymphoblastic leukemia. Haematologica 90: 1116-1127, 2005.

18. Westbrook CA, Hooberman AL, Spino C, Dodge RK, Larson RA, Davey F, Wurster-Hill DH, Sobol RE, Schiffer C and Bloomfield CD: Clinical significance of the BCR-ABL fusion gene in adult acute lymphoblastic leukemia: A cancer and leukemia group B study (8762). Blood 80: 2983-2990, 1992. 